OPEN ACCESS

Edited by:

Yimin Zhu,

Zhejiang University, China

Reviewed by:

Silvia Vannuccini,

University of Florence, Italy

Fan Jin,

Zhejiang University, China

${ }^{*}$ Correspondence:

Chao-qin Yu

chqyu81@163.com

Yan-ping Kuang

kuangyp@sh9hospital.org

${ }^{\dagger}$ These authors have contributed equally to this work

Specialty section: This article was submitted to

Reproduction,

a section of the journal

Frontiers in Endocrinology

Received: 07 December 2021 Accepted: 07 February 2022

Published: 08 March 2022

Citation:

Ni Z-x, Wan K-m, Zhou Z-h,

Kuang Y-p and Yu C-q (2022)

Impact of Maternal Age on

Singleton Birthweight in Frozen

Embryo Transfer Cycles.

Front. Endocrinol. 13:830414. doi: 10.3389/fendo.2022.830414

\section{Impact of Maternal Age on Singleton Birthweight in Frozen Embryo Transfer Cycles}

\author{
Zhe-xin $\mathrm{Ni}^{1 \dagger}$, Kun-ming $\mathrm{Wan}^{1+}$, Zhi-hao Zhou ${ }^{1+}$, Yan-ping Kuang ${ }^{2 *}$ and Chao-qin $\mathrm{Yu}^{1 *}$ \\ 1 Department of Traditional Chinese Medicine Gynecology, Changhai Hospital, Naval Medical University, Shanghai, China, \\ 2 Department of Assisted Reproduction, Shanghai Ninth People's Hospital, Shanghai JiaoTong University School of \\ Medicine, Shanghai, China
}

Background: Previous studies have investigated the effect of maternal age on assisted reproductive technology success rates. However, little is known about the relationship between maternal age and neonatal birthweight in frozen embryo transfer (FET) cycles. Whether maternal age influences singleton birthweight in FET cycles remains to be elucidated.

Methods: This study was conducted at a tertiary care center, involving singleton live births born to women undergoing frozen-thawed embryo transfer during the period from January 2010 to December 2017. A total of 12,565 women who fulfilled the inclusion criteria were enrolled and grouped into four groups according to the maternal age: $<30$, 30-34, 35-39, and $\geq 40$ years old. A multivariable linear regression analysis was conducted to reveal the relationship between maternal age and neonatal birthweight with controlling for a number of potential confounders.

Results: The highest proportions of low birthweight (LBW, 4.1\%), high birthweight (1.2\%), preterm birth (PTB, 5.9\%), and very PTB (0.9\%) were found in the group over 40 years old, but no significant difference was observed among the four groups. Additionally, the 3539-year-old group had the highest rate of very LBW (0.6\%), whereas the 30-34-year-old group had the lowest rate of small for gestational age (SGA, 2.7\%). However, multivariate analyses revealed that neonatal outcomes including PTB, LBW, and SGA were similar between the different maternal age groups.

Conclusion: Grouping with different maternal age was not associated with mean birthweight and Z-scores of singletons resulting from FET.

Keywords: assisted reproductive technology, frozen-thawed embryo transfer, vitrification, maternal age, birthweight

\section{BACKGROUND}

In the past decades, many women delay childbearing until after the age of 40 . The decline of fertility of elderly women forced them to seek the help of assisted reproductive technology (ART). According to statistics by the European IVF-monitoring Consortium, the proportion of women over 40 years of age that became pregnant through in vitro fertilization or intracytoplasmic sperm 
injection (IVF/ICSI) in Europe is increasing annually. For example, in the UK, the proportion increased from $12.7 \%$ to $26 \%$ in 1997 to 2015, and the same trend was observed in other high-income countries $(1,2)$.

Having a healthy baby is the ultimate goal of ART treatment. Increased risk of adverse neonatal outcomes is believed to appear in IVF/ICSI-conceived babies, even singletons, such as preterm birth (PTB), low birthweight (LBW), and small for gestational age (SGA), when compared with babies that are conceived spontaneously (3). Maternal age is a frequently used predictor of PTB, LBW, and perinatal mortality in women who have conceived naturally (4-7), and a number of studies have well revealed the negative correlation between maternal age and neonatal outcomes $(8,9)$. Women who have a baby through ART are, on average, older than those who conceive spontaneously as these techniques are often applied in response to age-related infertility problems (10). However, little is known regarding the influence of maternal age on birthweight in vitrified-thawed embryo transfer cycles.

To our knowledge, only few studies have examined the effect of maternal age on birthweight with ART, included different kinds of treatments, and considered sufficient confounders (1114). Furthermore, the published data, except for Lin's study (14), exclusively focused on fresh IVF cycles, without ruling out the possibility of adverse fetal growth caused by a hypoestrogenic milieu. Of note, supraphysiological estrogen levels during ovarian stimulation can create a suboptimal peri-implantation environment for implantation and placentation, thus causing abnormal fetal growth including LBW and SGA $(15,16)$. Unlike fresh ovarian stimulation cycles, frozen embryo transfer (FET) seems to provide a more physiological uterine environment for early fetal development (17). Thus, the current study aims to explore the effect of maternal age on the birthweight of newborns conceived through embryo transfers during FET cycles.

\section{METHODS}

\section{Study Design and Population}

This retrospective study involved women who had undergone FET during the period from January 2010 to December 2017, which was performed at the Department of Assisted Reproduction of the Ninth People's Hospital of Shanghai Jiao Tong University School of Medicine. Women who met the following inclusion criteria were included in the study: BMI $<30 \mathrm{~kg} / \mathrm{m}^{2}$ and transfer of embryos resulting in a live singleton birth. In all FET cycles, no more than two embryos can be transferred. Furthermore, only the first live birth IVF/ICSI cycles were retained for women who had more than one delivery during the study period. The exclusion criteria were as follows: vanishing twin syndrome, congenital uterine malformations, and presence of submucosal fibroids or polyps and intramural fibroids $>4 \mathrm{~cm}$ determined by ultrasound or hysteroscopy. Women with gestational diabetes, pregnancyinduced hypertension, and preeclampsia were excluded as these pregnancy-related factors may have a negative effect on intrauterine fetal growth. This study was approved by the institutional review board of the hospital and was conducted in accordance with the Helsinki Declaration. Informed consent was not required due to the retrospective nature of the study, and patients' data were used anonymously.

\section{Laboratory Protocols}

The procedures of ovarian stimulation, oocyte retrieval, and IVF/ ICSI have been described in previous studies $(18,19)$. In brief, IVF or ICSI was conventionally performed according to semen parameters and previous fertilization histories. For IVF, oocytes were inseminated in human tubal fluid (HTF, Irvine Scientific), which was supplemented with $10 \%$ serum substitute supplement (SSS, Irvine Scientific) and $\sim 300,000$ progressively motile spermatozoa. For ICSI, oocytes were transferred into dishes immediately after microinjection with $\mathrm{HTF}+10 \%$ SSS. The assessment of fertilization was performed $16-18 \mathrm{~h}$ after insemination/injection. A dish containing pre-equilibrated culture medium was then prepared for the transfer of zygotes. Before 2013, embryos were cultured in Early Cleavage Medium (Irvine Scientific) before day 3 and then in MultiBlast Medium (Irvine Scientific). However, a continuous single culture medium (Irvine Scientific) was introduced after January 2013. All embryos were cultured under mineral oil and grown in an incubator at $37^{\circ} \mathrm{C}$ under $5 \% \mathrm{O}_{2}$ and $6 \% \mathrm{CO}_{2}$ concentration (the balance gas was nitrogen). Except for the change of culture medium types, no change was made for other laboratory conditions and IVF protocols throughout the study period.

\section{Endometrial Preparation and Vitrification}

The endometrial preparation protocols for FET have been previously described (20). Briefly, a natural cycle FET was suitable for women having regular menstrual cycles with the use of HCG for triggering ovulation. Artificial cycles were offered for women with irregular cycles according to the discretion of treating physicians. The procedure of vitrification and thawing have been previously described (19). In short, the Cryotop carrier system with dimethyl sulfoxide-ethylene glycol-sucrose was used as cryoprotectants for embryo vitrification. Dilution solution in a sequential manner $(1 \mathrm{~mol} / \mathrm{L}$ to $0.5 \mathrm{~mol} / \mathrm{L}$ to 0 $\mathrm{mol} / \mathrm{L}$ sucrose) was used for thawing embryos. All embryos were thawed on the day of transfer.

\section{Maternal Age}

Maternal age at the birth of the child was the key explanatory variable, which was divided into the following categories: $<30$, $30-34,35-39$, and $\geq 40$ years old. The age group $<30$ years old was set as the reference category in our analyses.

\section{Outcome Measures}

The primary neonatal outcomes focused on live singleton birthweight, including Z-scores, birthweight categories, and birthweight percentiles. Secondary outcome measures were associated with neonatal outcomes, including GA at birth and newborn gender. The definition for live singleton birth was a delivery of a singleton viable infant after the 24th gestational week. GA in FET cycles was calculated from the day of embryo transfer (day 17 for cleavage-stage embryo transfer and day 19 for blastocyst embryo transfer) (21). The definitions for PTB and 
very PTB were live births at $<37$ and $<32$ completed gestational weeks, respectively. Z-scores were calculated according to GA and newborn gender on birthweight based on the national birthweight reference as previously described $(22,23)$. A birth weight $<2500 \mathrm{~g}$ was defined as LBW, and that $<1500 \mathrm{~g}$ was defined as very LBW. The birthweight of infant was divided as follows: LBW $(<2500 \mathrm{~g})$, very LBW (<1500 g), high birthweight (HBW, >4500 g), and normal birthweight. Birthweight percentiles were also based on the national birthweight reference (23) and were divided into the following categories: SGA defined as birthweight $<10$ th percentile, very SGA defined as birthweight $<3$ rd percentile, large for gestational age (LGA) defined as birthweight $>90$ th percentile, and very LGA defined as birthweight $>97$ th percentile.

\section{Statistical Analysis}

One-way analysis of variance was performed for continuous data, whereas Pearson's chi-squared test or Fisher's exact test was applied for categorical data. A post hoc Bonferroni correction was performed for multiple comparisons. The association between maternal age and neonatal outcomes was detected by multivariable logistic regression analysis, and the independent effect of maternal age on neonatal outcomes was analyzed by multiple linear regression.

The multivariable analyses included the following confounders: maternal BMI, paternal age, parity, infertility cause and duration, insemination method, type of endometrial preparation, endometrial thickness, year of treatment, and newborn gender. The continuous covariates (maternal BMI, paternal age, infertility duration, endometrial thickness, and year of treatment) and categorical covariates in multivariable models are listed in Table $\mathbf{1}$. Maternal age $<30$ years old was taken as a reference group in multivariable analyses. For the development of IVF techniques over time (24), a sensitivity analysis was performed on treating the year of treatment as a categorical variable. All analyses were conducted with SPSS Statistics (version 21.0), and $P<0.05$ was considered to be statistically significant.

\section{RESULTS}

The final dataset included 12,565 women who fulfilled the inclusion criteria, with no loss to follow-up. Baseline demographic and cycle characteristics are presented in Table 1. Comparison between the reference group and the other three groups revealed significant difference for maternal BMI, infertility cause, parity, infertility duration, FET cycle rank, fertilization method, number of embryo transferred, FET endometrial preparation, endometrial thickness, and year of treatment. Infertility duration and embryo developmental stage at transfer did not differ significantly among maternal age categories.

Neonatal outcomes stratified by maternal age are listed in Table 2. No significant difference was observed on GA and mean birthweight, and the gestation-adjusted Z-scores varied significantly according to maternal age categories. The 30-34year-old group had the highest birthweight and Z-scores (3355.8 \pm $483.3 \mathrm{~g}, 0.38 \pm 1.03$ ). With the increase of maternal age, a modest decrease of birthweight was observed, and the group over 40 years old had the lowest birthweight values (3321.6 $\pm 503.9 \mathrm{~g})$. Additionally, no significant differences were found between any two groups by post hoc analysis on birthweight and Z-scores. The highest proportions of LBW (4.1\%), HBW (1.2\%), PTB (5.9\%), and very PTB (0.9\%) were found in the group over 40 years old, but no difference was observed among the four groups. Interestingly, the 35-39-year-old group had the highest rate of very LBW $(0.6 \%)$, whereas the 30-34-year-old group had the lowest rate of SGA (2.7\%). Furthermore, no difference was observed in very LGA, LGA, very SGA, and newborn gender between groups.

In multivariate analyses (Table 3 ), the neonatal outcomes including PTB, LBW, HBW, SGA, and LGA were similar between the different maternal age groups. The odds of PTB and LBW were lower in the group over 40 years old compared with the reference group, which did not reach a significant difference. Although the analysis of very PTB ( $<32$ weeks) and very LBW $(<1500 \mathrm{~g})$ was performed, the number of cases in the two categories was too small to make any meaningful comparisons. In addition, no significant difference was found on birthweight percentile categories between the reference group and the other three groups.

Multiple linear regression analyses were conducted to assess the relationship between maternal age and birthweight (Table 4). Even after correction for a number of potential confounders, no significant correlation was found between maternal age and neonatal birthweight. Moreover, maternal BMI $(P<0.001)$, parity $(P<0.001)$, FET cycle rank $(P=0.001)$, number of embryos transferred $(P=0.034)$, embryo developmental stage at transfer $(P<$ $0.001)$, endometrial thickness $(8-11 \mathrm{~mm}, P=0.018$; $>11 \mathrm{~mm}, P=$ $0.001)$, year of treatment $(P<0.001)$, GA $(P<0.001)$, and newborn gender $(P<0.001)$ were all independent predictors for birthweight.

\section{DISCUSSION}

In recent years, much attention has been paid to the impact of maternal age on ART success rates, and several studies have revealed that a high maternal age (over 40 years old) has a negative effect on pregnancy outcomes $(11,13)$. However, our study showed that no significant association existed between maternal age and singleton birthweight in FET cycles with consideration for related confounders. Furthermore, linear regression indicated that maternal age was not an independent predictor of singleton birthweight in FET cycles.

To our knowledge, four studies have analyzed the potential relationship between maternal age and neonatal birthweight. Wennberg et al. investigated the influence of maternal age on adverse maternal and neonatal outcomes following ART treatment and found that the risk of LBW and very LBW was significantly higher in ART than in spontaneous conception (SC) singletons in all ages up to maternal age of 40 years (LBW: aORs 
TABLE 1 | Patient treatment and demographic characteristics according to maternal age.

\begin{tabular}{|c|c|c|c|c|c|c|c|}
\hline & $\begin{array}{c}<30 y \\
n=3586\end{array}$ & $\begin{array}{l}30-34 y \\
n=5461\end{array}$ & $\begin{array}{l}35-39 y \\
n=2861\end{array}$ & $\begin{array}{c}\geq 40 y \\
n=657\end{array}$ & P value ${ }^{a}$ & $P$ value $^{b}$ & P value ${ }^{c}$ \\
\hline Maternal Age (years) & $27.29 \pm 1.63$ & $31.93 \pm 1.40$ & $36.47 \pm 1.34$ & $41.31 \pm 1.53$ & $<0.001$ & $<0.001$ & $<0.001$ \\
\hline Maternal BMI (kg/m2) & & & & & $<0.001$ & $<0.001$ & $<0.001$ \\
\hline$<18.5$ & $534(14.9)$ & 615 (11.3) & $270(9.4)$ & $37(5.6)$ & & & \\
\hline 18.5-22.9 & 2218 (61.9) & $3448(63.1)$ & $1791(62.6)$ & $414(63.0)$ & & & \\
\hline 23-27.4 & 729 (20.3) & $1254(23.0)$ & $719(25.1)$ & $194(29.5)$ & & & \\
\hline$\geq 27.5$ & $105(2.9)$ & $144(2.6)$ & $81(2.8)$ & $12(1.8)$ & & & \\
\hline Paternal Age (years) & $29.69 \pm 3.53$ & $33.74 \pm 3.68$ & $38.03 \pm 4.29$ & $42.62 \pm 5.15$ & $<0.001$ & $<0.001$ & $<0.001$ \\
\hline Infertility cause & & & & & $<0.001$ & $<0.001$ & $<0.001$ \\
\hline Tubal factors & 2126 (59.3) & 3226 (59.1) & $1788(62.5)$ & $321(48.9)$ & & & \\
\hline PCOS & 391 (10.9) & $425(7.8)$ & $97(3.4)$ & $12(1.8)$ & & & \\
\hline Endometriosis & $255(7.1)$ & $466(8.5)$ & $280(9.8)$ & $57(8.7)$ & & & \\
\hline Diminished ovarian reserve. & $61(1.7)$ & $121(2.2)$ & $117(4.1)$ & $142(21.6)$ & & & \\
\hline Uterine factors & $52(1.5)$ & $112(2.1)$ & 65 (2.3) & $17(2.6)$ & & & \\
\hline Male & $513(14.3)$ & $675(12.4)$ & $294(10.3)$ & $67(10.2)$ & & & \\
\hline Unexplained & $188(5.2)$ & $436(8.0)$ & $220(7.7)$ & $41(6.2)$ & & & \\
\hline Parity & & & & & $<0.001$ & $<0.001$ & $<0.001$ \\
\hline 0 & 3489 (97.3) & $5159(94.5)$ & 2478 (86.6) & $471(71.7)$ & & & \\
\hline$>0$ & $97(2.7)$ & $302(5.5)$ & $383(13.4)$ & $186(28.3)$ & & & \\
\hline Infertility duration (years) & $2.53 \pm 1.82$ & $3.29 \pm 2.42$ & $4.11 \pm 3.41$ & $4.70 \pm 4.55$ & 0.058 & 0.067 & 0.11 \\
\hline FET cycle rank & & & & & $<0.001$ & $<0.001$ & $<0.001$ \\
\hline First & $2332(65.0)$ & $3112(57.0)$ & $1444(50.5)$ & $298(45.4)$ & & & \\
\hline High order & $1254(35.0)$ & $2349(43.0)$ & $1417(49.5)$ & $359(54.6)$ & & & \\
\hline Fertilization method & & & & & 0.118 & $<0.001$ & $<0.001$ \\
\hline IVF & $2210(61.6)$ & $3425(62.7)$ & $1871(65.4)$ & $414(63.0)$ & & & \\
\hline ICSI & $990(27.6)$ & 1407 (25.8) & 762 (26.6) & $225(34.2)$ & & & \\
\hline$|\mathrm{VF}+| \mathrm{CSI}$ & $386(10.8)$ & $629(11.5)$ & $228(8.0)$ & $18(2.7)$ & & & \\
\hline Number of embryos transferred & & & & & 0.002 & $<0.001$ & $<0.001$ \\
\hline 1 & $555(15.5)$ & 985 (18.0) & 569 (19.9) & $145(22.1)$ & & & \\
\hline$\geq 2$ & 3031 (84.5) & $4476(82.0)$ & $2292(80.1)$ & $512(77.9)$ & & & \\
\hline Embryo developmental stage at transfer & & & & & 0.815 & 0.607 & 0.200 \\
\hline Day 3 & 3010 (83.9) & $4573(83.7)$ & 2415 (84.4) & $565(86.0)$ & & & \\
\hline Day $5 / 6$ & $576(16.1)$ & $888(16.3)$ & $446(15.6)$ & $92(14.0)$ & & & \\
\hline FET endometrial preparation & & & & & $<0.001$ & $<0.001$ & $<0.001$ \\
\hline Natural cycle & 737 (20.6) & $1338(24.5)$ & $750(26.2)$ & $176(26.8)$ & & & \\
\hline Artificial cycle & 2849 (79.4) & $4123(75.5)$ & $2111(73.8)$ & $481(73.2)$ & & & \\
\hline Endometrial thickness (mm) & & & & & 0.040 & $<0.001$ & $<0.001$ \\
\hline$<8$ & $232(6.5)$ & $399(7.3)$ & $270(9.4)$ & $83(12.6)$ & & & \\
\hline $8-11$ & 1958 (54.6) & 3067 (56.2) & 1610 (56.3) & $369(56.2)$ & & & \\
\hline$>11$ & 1396 (38.9) & 1995 (36.5) & $981(34.3)$ & $205(31.2)$ & & & \\
\hline Year of treatment & & & & & 0.380 & 0.001 & $<0.001$ \\
\hline 2010-2012 & $276(7.7)$ & 465 (8.5) & $218(7.6)$ & $33(5.0)$ & & & \\
\hline 2013-2014 & 1693 (47.2) & 2561 (46.9) & 1219 (42.6) & $250(38.1)$ & & & \\
\hline $2015-2017$ & $1617(45.1)$ & 2435 (44.6) & $1424(49.8)$ & $375(56.9)$ & & & \\
\hline
\end{tabular}

a30-34 years old $v$ s. $<30$ years old.

${ }^{b} 35-39$ years old $v$ s. $<30$ years old.

${ }^{c} \geq 40$ years old $v$ s. $<30$ years old.

Data are presented as mean $\pm S D$ for continuous variables and $n$ (\%) for dichotomous variables.

1.44-2.35; VLBW: aORs 1.67-3.44). Additionally, when the analysis was restricted to maternal age $>35$ years, an increased risk of LBW existed for SC pregnancies, but not for ART pregnancies (11). Due to medical, educational, and socioeconomic reasons, women aged $>35$ years who conceive through ART may pay more attention to their state of health and seek medical assistance more often than SC women, which could result in increased detection of complications and decreased risk of LBW. Second, Moaddab et al. found that maternal age did not predict newborns' birthweight in pregnancies with maternal age grouping as $<40,40-44,45-49$, and $\geq 50$ years old (12). However, the analysis on birthweight in the maternal age group $<40$ years old per se is inadequate, and important information is missing. There may be some interesting findings among groups at ages $<25,26-30,31-34$, and $35-40$ years old, and the increased risk of LBW may appear at the age of 35 years old. However, this study seemed to miss this potential information. Many studies have set up more detailed groups with maternal age under 40 years old to assess the influence of maternal age on neonatal outcomes and gained more credible results $(13,14)$. Another study reported that the risk of LBW was increased only at maternal ages over 40 years old (6 percentage points, $95 \%$ CI: $0.2,12)$ with medically assisted reproduction (MAR) compared with the risk of LBW at ages 30-34 years old (13). However, a limited number of 
TABLE 2 | Neonatal outcomes of live born singletons by maternal age.

\begin{tabular}{|c|c|c|c|c|c|c|c|}
\hline & $\begin{array}{c}<30 y \\
n=3586\end{array}$ & $\begin{array}{l}30-34 y \\
n=5461\end{array}$ & $\begin{array}{l}35-39 y \\
n=2861\end{array}$ & $\begin{array}{c}\geq 40 y \\
n=657\end{array}$ & P value ${ }^{a}$ & P value ${ }^{b}$ & P value ${ }^{c}$ \\
\hline Gestational age & & & & & 0.779 & 0.324 & 0.487 \\
\hline$\geq 37$ weeks & 3367 (93.9) & $5117(93.7)$ & 2694 (94.2) & $612(93.2)$ & & & \\
\hline preterm birth (<37 weeks) & $198(5.5)$ & $316(5.8)$ & $143(5.0)$ & $39(5.9)$ & & & \\
\hline very preterm birth (<32 weeks) & $21(0.6)$ & $28(0.5)$ & $24(0.8)$ & $6(0.9)$ & & & \\
\hline Birthweight (g) & $3352.2 \pm 485.7$ & $3355.8 \pm 483.3$ & $3334.2 \pm 493.6$ & $3321.6 \pm 503.9$ & 0.976 & 0.336 & 0.335 \\
\hline Z-scores & $0.36 \pm 1.04$ & $0.38 \pm 1.03$ & $0.35 \pm 1.06$ & $0.38 \pm 1.10$ & 0.754 & 0.984 & 0.939 \\
\hline Birthweight categories & & & & & 0.761 & 0.464 & 0.240 \\
\hline Very low birthweight (<1500 g) & $12(0.3)$ & $19(0.3)$ & $17(0.6)$ & $2(0.3)$ & & & \\
\hline Low birthweight (<2500 g) & $107(3.0)$ & $181(3.3)$ & $90(3.1)$ & $27(4.1)$ & & & \\
\hline High birthweight (>4500 g) & $27(0.8)$ & $48(0.9)$ & $22(0.8)$ & $8(1.2)$ & & & \\
\hline Birthweight percentiles & & & & & 0.186 & 0.498 & 0.937 \\
\hline Very small for gestational age (<3rd percentile) & $48(1.3)$ & $83(1.5)$ & $35(1.2)$ & $9(1.4)$ & & & \\
\hline Small for gestational age (<10th percentile) & $126(3.5)$ & $150(2.7)$ & $100(3.5)$ & $20(3.0)$ & & & \\
\hline Large for gestational age (>90th percentile) & $369(10.3)$ & $583(10.7)$ & $326(11.4)$ & $74(11.3)$ & & & \\
\hline Very large of gestational age (>97th percentile) & $258(7.2)$ & $354(6.5)$ & $183(6.4)$ & $46(7.0)$ & & & \\
\hline Newborn gender & & & & & 0.667 & 0.564 & 0.393 \\
\hline Female & $1881(52.5)$ & $2839(52.0)$ & $1522(53.2)$ & $349(53.1)$ & & & \\
\hline Male & $1705(47.5)$ & $2622(48.0)$ & $1339(46.8)$ & 308 (46.9) & & & \\
\hline
\end{tabular}

a30-34 years old $v$ s. $<30$ years old.

${ }^{b} 35-39$ years old $v$ s. $<30$ years old.

${ }^{c} \geq 40$ years old $v s$. $<30$ years old.

Data are presented as mean $\pm S D$ for continuous variables and $n(\%)$ for dichotomous variables.

TABLE 3 | Odds ratios for gestational age and birth weights by maternal age.

\begin{tabular}{|c|c|c|c|c|}
\hline & $\begin{array}{c}<30 y \\
n=3586\end{array}$ & $\begin{array}{l}30-34 y \\
n=5461\end{array}$ & $\begin{array}{l}35-39 y \\
n=2861\end{array}$ & $\begin{array}{l}\geq 40 y \\
n=657\end{array}$ \\
\hline \multicolumn{5}{|c|}{ Gestational age between $<37$ weeks } \\
\hline Adjusted OR & Reference & $0.951(0.749-1.209)$ & $1.096(0.779-1.542)$ & $0.947(0.586-1.530)$ \\
\hline \multicolumn{5}{|c|}{ Gestational age $<32$ weeks } \\
\hline Crude OR & Reference & $1.140(0.646-2.010)$ & $0.700(0.389-1.260)$ & $0.636(0.256-1.583)$ \\
\hline Adjusted OR & Reference & $1.015(0.476-2.163)$ & $0.825(0.301-2.257)$ & $0.741(0.206-2.668)$ \\
\hline Adjusted OR & Reference & $1.132(0.470-2.728)$ & $0.701(0.222-2.217)$ & $1.211(0.203-7.228)$ \\
\hline \multicolumn{5}{|c|}{ Low birthweight (<2500 g) } \\
\hline Crude OR & Reference & $0.896(0.703-1.142)$ & $0.944(0.710-1.255)$ & $0.715(0.465-1.100)$ \\
\hline Adjusted OR & Reference & $0.959(0.703,1.309)$ & $1.029(0.662-1.599)$ & $0.787(0.435-1.423)$ \\
\hline \multicolumn{5}{|c|}{ High birthweight (>4500 g) } \\
\hline Crude OR & Reference & $0.836(0.521-1.339)$ & $0.975(0.554-1.716)$ & $0.610(0.276-1.350)$ \\
\hline \multicolumn{5}{|c|}{ Small for gestational age ( $<10$ th percentile) } \\
\hline Crude OR & Reference & $1.294(1.016-1.648)$ & $1.003(0.767-1.312)$ & $1.149(0.710-1.859)$ \\
\hline Adjusted OR & Reference & $1.217(0.877-1.260)$ & $1.015(0.655-1.573)$ & $1.066(0.564-2.017)$ \\
\hline \multicolumn{5}{|c|}{ Large for gestational age (>90th percentile) } \\
\hline Crude OR & Reference & $0.975(0.849-1.120)$ & $0.901(0.769-1.056)$ & $0.910(0.696-1.188)$ \\
\hline Adjusted OR & Reference & $1.051(0.877-1.260)$ & $1.026(0.799-1.316)$ & $1.034(0.721-1.483)$ \\
\hline \multicolumn{5}{|c|}{ Very large of gestational age ( $>97 \mathrm{th}$ percentile) } \\
\hline Crude OR & Reference & $1.123(0.950-1.328)$ & $1.122(0.921-1.367)$ & $1.023(0.737-1.420)$ \\
\hline Adjusted OR & Reference & $1.071(0.856-1.339)$ & $1.052(0.769-1.439)$ & $0.983(0.623-1.549)$ \\
\hline
\end{tabular}


TABLE 4 | Results of multiple regression analysis of singleton birthweight.

\begin{tabular}{|c|c|c|c|c|c|}
\hline & \multirow{2}{*}{$\frac{\text { Unstandardized coefficients }}{\text { B }}$} & \multirow[t]{2}{*}{ Std. error } & \multirow{2}{*}{$\frac{\text { Standardized coefficients }}{\text { Beta }}$} & \multirow[t]{2}{*}{$\mathbf{t}$} & \multirow[t]{2}{*}{ P value } \\
\hline & & & & & \\
\hline (Constant) & -3532.855 & 99.754 & & -35.416 & $<0.001$ \\
\hline \multicolumn{6}{|l|}{ Maternal age } \\
\hline \multicolumn{6}{|l|}{$<30$ (reference) } \\
\hline $30-34$ & -4.313 & 9.513 & -0.004 & -0.453 & 0.650 \\
\hline 35-39 & -14.879 & 13.020 & -0.013 & -1.143 & 0.253 \\
\hline$\geq 40$ & -13.387 & 21.706 & -0.006 & -0.617 & 0.537 \\
\hline \multicolumn{6}{|l|}{ Maternal BMI (kg/m²) } \\
\hline$<18.5$ & -109.739 & 11.516 & -0.072 & -9.530 & $<0.001$ \\
\hline \multicolumn{6}{|l|}{ 18.5-22.9 (reference) } \\
\hline 23-27.4 & 86.312 & 8.825 & 0.075 & 9.781 & $<0.001$ \\
\hline$\geq 27.5$ & 157.905 & 22.417 & 0.053 & 7.044 & $<0.001$ \\
\hline Paternal age & 1.556 & 0.932 & 0.017 & 1.669 & 0.095 \\
\hline Parity, high order (versus 0 ) & 62.961 & 13.955 & 0.034 & 4.512 & $<0.001$ \\
\hline Infertility duration (years) & 1.282 & 1.364 & 0.007 & 0.940 & 0.347 \\
\hline FET cycle rank, High order (versus First) & 26.116 & 7.516 & 0.027 & 3.474 & 0.001 \\
\hline \multicolumn{6}{|l|}{ Infertility cause } \\
\hline \multicolumn{6}{|l|}{ Tubal factors (reference) } \\
\hline PCOS & 9.216 & 14.497 & 0.005 & 0.636 & 0.525 \\
\hline Endometriosis & 2.194 & 13.273 & 0.001 & 0.165 & 0.869 \\
\hline Diminished ovarian reserve. & -10.895 & 20.651 & -0.004 & -0.528 & 0.598 \\
\hline Uterine factors & -10.179 & 26.186 & -0.003 & -0.389 & 0.697 \\
\hline Male & -14.409 & 12.591 & -0.010 & -1.144 & 0.252 \\
\hline Unexplained & -7.991 & 14.833 & -0.004 & -0.539 & 0.590 \\
\hline \multicolumn{6}{|l|}{ Fertilization method } \\
\hline \multicolumn{6}{|l|}{ IVF (reference) } \\
\hline ICSI & -18.255 & 9.320 & -0.017 & -1.959 & 0.050 \\
\hline VF+lCSI & 4.260 & 12.769 & 0.003 & 0.334 & 0.739 \\
\hline Number of embryos transferred, $\geq 2$ (versus 1 ) & 22.384 & 10.540 & 0.018 & 2.124 & 0.034 \\
\hline Embryo developmental stage at transfer, Day 5/6 (versus Day 3) & 71.030 & 11.139 & 0.053 & 6.377 & $<0.001$ \\
\hline $\begin{array}{l}\text { FET endometrial preparation, Artificial cycle (versus Natural } \\
\text { cycle) }\end{array}$ & -15.143 & 8.558 & -0.013 & -1.769 & 0.077 \\
\hline \multicolumn{6}{|l|}{ Endometrial thickness (mm) } \\
\hline \multicolumn{6}{|l|}{$<8$ (reference) } \\
\hline $8-11$ & 32.475 & 13.757 & 0.033 & 2.361 & 0.018 \\
\hline$>11$ & 45.989 & 14.243 & 0.045 & 3.229 & 0.001 \\
\hline \multicolumn{6}{|l|}{ Year of treatment } \\
\hline \multicolumn{6}{|l|}{ 2010-2012 (reference) } \\
\hline 2013-2015 & -61.556 & 13.940 & -0.063 & -4.416 & $<0.001$ \\
\hline 2016-2017 & -77.498 & 14.127 & -0.079 & -5.486 & $<0.001$ \\
\hline Gestational age (week) & 178.687 & 2.429 & 0.548 & 73.556 & $<0.001$ \\
\hline Newborn gender, female (versus male) & -137.516 & 7.200 & -0.141 & -19.098 & $<0.001$ \\
\hline
\end{tabular}

confounders were included in the study, and the effect of different kinds of MAR treatments could not be reliably distinguished, which included less invasive treatments such as ovulation induction only that were less strongly associated with adverse birth outcomes (25). A recent study based on 4958 infertile women using a freeze-all strategy observed that maternal age grouping was not related with increased risks of LBW, very LBW, preterm LBW, and macrosomia (14). However, only 1450 singleton live births were involved for the analysis of LBW, and the 44-50-year-old group of singleton live births was very small $(n=9)$, which may limit the power of statistical analyses between groups.

Our study aimed to improve on the flaws of the abovementioned studies and focused on the exact role of maternal age in singleton birthweight after FET cycles. The current study based on 12,565 singleton newborns born after
FET cycles demonstrated that maternal age itself had no impact on singleton birthweight, and neonatal outcomes including PTB, LBW, and SGA were similar between the different maternal age groups. Due to several confounding factors, direct comparability across different age groups has very limited clinical significance in Table 2. Because of the strict exclusion criteria, the generality of this finding may be, to some extent, restricted. The reason why no significant correlation existed between maternal age and birthweight in our study is likely complex. It is generally known that with aging comes a reduction in ovarian function, resulting in the decrease of ovarian response to ovulationpromoting drugs and the low number of oocytes retrieved (26). Additionally, the decreased quality of oocytes $(27,28)$, abnormal endometrial function, and degeneration of multiple organ function will appear in women with advanced maternal age $(22,29)$. All the above-mentioned factors would affect the 
development of the embryo and cause adverse effects on the newborn, leading to LBW. However, with the popularization of education, many women tend to choose late marriage and late childbearing and enjoy a simple single life before marriage. In this kind of life, they are less stressed and have more opportunities to get in touch with life than women who are married and have children. Meanwhile, these knowledgeable women tend to choose a healthy and regular life and possess good habits, physical quality, and economic conditions, thus having a better choice on ART treatment (11). Aging leads to an irreversible decline in fertility, forcing older women to pay more attention to pregnancy and to seek medical help more actively than young women. In addition, the spouse's income increases with age to guarantee maternity. Most importantly, the development of ART has well fulfilled the reproductive needs of women with different ages to improve the quality of newborns.

In this study, the results of multiple linear regression analysis indicated that maternal BMI, embryo developmental stage at transfer, parity, number of embryos transferred, endometrial thickness, year of treatment, GA, and newborn gender were independent predictors for neonatal birthweight, which was consistent with previous results $(22,30,31)$. Z-scores were calculated and compared across the four groups to reduce bias caused by newborn gender and GA, and no significant difference on Z-scores was found among different maternal age groups. In addition, significant differences were observed between the maternal age groups in baseline and cycle characteristics including infertility duration, infertility cause, and fertilization method. However, these confounders had no impact on neonatal birthweight based on the linear regression model.

This study has several limitations. The biggest one is its retrospective design, so we strictly checked the database with strict criteria. Second, due to personal privacy restrictions, we were unable to obtain the education and economic background of patients. Third, many confounding factors were strongly associated with birthweight in linear regression analysis. Data bias during the experimental design cannot be all corrected by regression equations. Furthermore, embryo quality and paternal BMI were important factors that may affect neonatal outcomes, yet these data were missed in this study. However, the large number of singleton live births from a single center can assure the practice consistency, which is the main strength of the current study. Additionally, aside from the change of culture medium types, all other laboratory conditions and protocols remained invariant throughout the study period. Furthermore, maternal age was recorded according to the identification card, and endometrial thickness was measured by the same trained sonographers, reducing recorder variability. Importantly, a number of potential confounders were included in our study, which may minimize their impact on the findings.

\section{REFERENCES}

1. Nygren KG, Andersen AN. Assisted Reproductive Technology in Europe, 1997. Results Generated From European Registers by ESHRE. European IVFMonitoring Programme (EIM), for the European Society of Human

\section{CONCLUSIONS}

This study expands the current knowledge about the association between maternal age and neonatal outcomes and shows that maternal age is not associated with mean birthweight and Zscores. This important finding should be adequately applied for women over 40 years old prior to FET and strengthen their confidence. A large prospective study, of course, is needed to verify our findings in the future.

\section{DATA AVAILABILITY STATEMENT}

The original contributions presented in the study are included in the article/supplementary material. Further inquiries can be directed to the corresponding authors.

\section{ETHICS STATEMENT}

This study was approved by the institutional review board of the Ninth People's Hospital of Shanghai Jiao Tong University School of Medicine (reference number 2017-211) and the Changhai Hospital of Naval Medical University (reference number CHEC 2019-100), and was carried out in accordance with the Helsinki Declaration. Due to the retrospective nature, informed consent was not required, and patients' data were used anonymously.

\section{AUTHOR CONTRIBUTIONS}

$\mathrm{C}-\mathrm{qY}$ and $\mathrm{Y}-\mathrm{pK}$ conceived and designed this study. Z-xN, K-mW, and $\mathrm{Z}$-hZ contributed to data acquisition, analysis and interpretation, and drafted the manuscript. K-mW and $\mathrm{Z}-\mathrm{xN}$ were responsible for the collection of data. All authors interpreted the data. All authors contributed to the article and approved the submitted version.

\section{FUNDING}

This work was supported by the National Natural Science Foundation of China (81774352, 81703874).

\section{ACKNOWLEDGMENTS}

The authors thank the nurses and laboratory staff of the Department of Assisted Reproduction for their contribution to this work. Moreover, the authors thank the infertile couples who participated in this study.

Reproduction and Embryology (ESHRE). Hum Reprod (2001) 16(2):38491. doi: 10.1093/humrep/16.2.384

2. De Geyter C, Calhaz-Jorge C, Kupka MS, Wyns C, Mocanu E, Motrenko T, et al. European IVF-Monitoring Consortium (EIM) for the European Society of Human Reproduction and Embryology (ESHRE). ART in Europe, 2015: 
Results Generated From European Registries by ESHRE. Hum Reprod Open (2020) 2020(1):hoz038. doi: 10.1093/hropen/hoz038

3. Berntsen S, Söderström-Anttila V, Wennerholm UB, Laivuori H, Loft A, Oldereid NB, et al. The Health of Children Conceived by ART: 'The Chicken or the Egg? Hum Reprod Update (2019) 25(2):137-58. doi: 10.1093/humupd/ dmz001

4. Kawwass JF, Badell ML. Maternal and Fetal Risk Associated With Assisted Reproductive Technology. Obstet Gynecol (2018) 132(3):763-72. doi: 10.1097/AOG.0000000000002786

5. Pinheiro RL, Areia AL, Mota Pinto A, Donato H. Advanced Maternal Age: Adverse Outcomes of Pregnancy, A Meta-Analysis. Acta Med Port (2019) 32 (3):219-26. doi: 10.20344/amp.11057

6. Leader J, Bajwa A, Lanes A, Hua X, Rennicks White R, Rybak N, et al. The Effect of Very Advanced Maternal Age on Maternal and Neonatal Outcomes: A Systematic Review. J Obstet Gynaecol Can (2018) 40(9):1208-18. doi: 10.1016/j.jogc.2017.10.027

7. Lean SC, Derricott H, Jones RL, Heazell AEP. Advanced Maternal Age and Adverse Pregnancy Outcomes: A Systematic Review and Meta-Analysis. PloS One (2017) 12(10):e0186287. doi: 10.1371/journal.pone.0186287

8. Wu Y, Chen Y, Shen M, Guo Y, Wen SW, Lanes A, et al. Adverse Maternal and Neonatal Outcomes Among Singleton Pregnancies in Women of Very Advanced Maternal Age: A Retrospective Cohort Study. BMC Pregnancy Childbirth (2019) 19(1):3. doi: 10.1186/s12884-018-2147-9

9. Koshida S, Arima H, Fujii T, Ito Y, Murakami T, Takahashi K. Impact of Advanced Maternal Age on Adverse Infant Outcomes: A Japanese Population-Based Study. Eur J Obstet Gynecol Reprod Biol (2019) 242:17881. doi: 10.1016/j.ejogrb.2019.08.011

10. Luke B, Brown MB. Elevated Risks of Pregnancy Complications and Adverse Outcomes With Increasing Maternal Age. Hum Reprod (2007) 22(5):1264-72. doi: 10.1093/humrep/del522

11. Wennberg AL, Opdahl S, Bergh C, Aaris Henningsen AK, Gissler M, Romundstad LB, et al. Effect of Maternal Age on Maternal and Neonatal Outcomes After Assisted Reproductive Technology. Fertil Steril (2016) 106 (5):1142-9.e14. doi: 10.1016/j.fertnstert.2016.06.021

12. Moaddab A, Chervenak FA, Mccullough LB, Sangi-Haghpeykar H, Shamshirsaz AA, Schutt A, et al. Effect of Advanced Maternal Age on Maternal and Neonatal Outcomes in Assisted Reproductive Technology Pregnancies. Eur J Obstet Gynecol Reprod Biol (2017) 216:178-83. doi: 10.1016/j.ejogrb.2017.07.029

13. Barbuscia A, Martikainen P, Myrskylä M, Remes H, Somigliana E, Klemetti R, et al. Maternal Age and Risk of Low Birth Weight and Premature Birth in Children Conceived Through Medically Assisted Reproduction. Evidence From Finnish Population Registers. Hum Reprod (2020) 35(1):212-20. doi: 10.1093/humrep/dez275

14. Lin J, Huang J, Zhu Q, Kuang Y, Cai R, Wang Y. Effect of Maternal Age on Pregnancy or Neonatal Outcomes Among 4,958 Infertile Women Using a Freeze-All Strategy. Front Med (Lausanne) (2020) 6:316. doi: 10.3389/ fmed.2019.00316

15. Pereira N, Elias RT, Christos PJ, Petrini AC, Hancock K, Lekovich JP, et al. Supraphysiologic Estradiol is an Independent Predictor of Low Birth Weight in Full-Term Singletons Born After Fresh Embryo Transfer. Hum Reprod (2017) 32(7):1410-7. doi: 10.1093/humrep/dex095

16. Imudia AN, Awonuga AO, Doyle JO, Kaimal AJ, Wright DL, Toth TL, et al. Peak Serum Estradiol Level During Controlled Ovarian Hyperstimulation is Associated With Increased Risk of Small for Gestational Age and Preeclampsia in Singleton Pregnancies After In Vitro Fertilization. Fertil Steril (2012) 97(6):1374-9. doi: 10.1016/j.fertnstert.2012.03.028

17. Järvelä IY, Pelkonen S, Uimari O, Mäkikallio K, Puukka K, Ruokonen A, et al. Controlled Ovarian Hyperstimulation Leads to High Progesterone and Estradiol Levels During Early Pregnancy. Hum Reprod (2014) 29(11):2393401. doi: 10.1093/humrep/deu223

18. Kuang Y, Chen Q, Hong Q, Lyu Q, Ai A, Fu Y, et al. Double Stimulations During the Follicular and Luteal Phases of Poor Responders in IVF/ICSI Programmes (Shanghai Protocol). Reprod BioMed Online (2014) 29(6):68491. doi: 10.1016/j.rbmo.2014.08.009

19. Kuang Y, Chen Q, Fu Y, Wang Y, Hong Q, Lyu Q, et al. Medroxyprogesterone Acetate is an Effective Oral Alternative for Preventing Premature Luteinizing
Hormone Surges in Women Undergoing Controlled Ovarian Hyperstimulation for In Vitro Fertilization. Fertil Steril (2015) 104(1):6270.e3. doi: $10.1016 /$ j.fertnstert.2015.03.022

20. Du T, Chen H, Fu R, Chen Q, Wang Y, Mol BW, et al. Comparison of Ectopic Pregnancy Risk Among Transfers of Embryos Vitrified on Day 3, Day 5, and Day 6. Fertil Steril (2017) 108(1):108-16.e1. doi: 10.1016/j.fertnstert.2017.05.027

21. Nelissen EC, Van Montfoort AP, Coonen E, Derhaag JG, Geraedts JP, Smits LJ, et al. Further Evidence That Culture Media Affect Perinatal Outcome: Findings After Transfer of Fresh and Cryopreserved Embryos. Hum Reprod (2012) 27(7):1966-76. doi: 10.1093/humrep/des145

22. Zhang J, Liu H, Mao X, Chen Q, Si J, Fan Y, et al. Effect of Endometrial Thickness on Birthweight in Frozen Embryo Transfer Cycles: An Analysis Including 6181 Singleton Newborns. Hum Reprod (2019) 34(9):1707-15. doi: 10.1093/humrep/dez103

23. Dai L, Deng C, Li Y, Zhu J, Mu Y, Deng Y, et al. Birth Weight Reference Percentiles for Chinese. PloS One (2014) 9(8):e104779. doi: 10.1371/ journal.pone. 0104779

24. Castillo CM, Horne G, Fitzgerald CT, Johnstone ED, Brison DR, Roberts SA. The Impact of IVF on Birthweight From 1991 to 2015: A Cross-Sectional Study. Hum Reprod (2019) 34(5):920-31. doi: 10.1093/humrep/dez025

25. De Geyter C, Calhaz-Jorge C, Kupka MS, Wyns C, Mocanu E, Motrenko T, et al. European IVF-Monitoring Consortium (EIM) for the European Society of Human Reproduction and Embryology (ESHRE). ART in Europe, 2014: Results Generated From European Registries by ESHRE: The European IVFMonitoring Consortium (EIM) for the European Society of Human Reproduction and Embryology (ESHRE). Hum Reprod (2018) 33(9):1586601. doi: 10.1093/humrep/dey242

26. Yang X, Zhang J, Wu J, Huang J, Chen Q, Lu X, et al. Association Between the Number of Oocytes Retrieved and Neonatal Outcomes After Freeze-All IVF Cycles. Hum Reprod (2019) 34(10):1937-47. doi: 10.1093/humrep/dez137

27. Pacella L, Zander-Fox DL, Armstrong DT, Lane M. Women With Reduced Ovarian Reserve or Advanced Maternal Age Have an Altered Follicular Environment. Fertil Steril (2012) 98(4):986-94.e1-2. doi: 10.1016/ j.fertnstert.2012.06.025

28. Pella R, Suarez-Cunza S, Orihuela P, Escudero F, Perez Y, Garcia M, et al. Oxidative Balance in Follicular Fluid of ART Patients of Advanced Maternal Age and Blastocyst Formation. JBRA assisted Reprod (2020) 24(3):296-301. doi: 10.5935/1518-0557.20200012

29. Gibson DA, Simitsidellis I, Kelepouri O, Critchley HOD, Saunders PTK. Dehydroepiandrosterone Enhances Decidualization in Women of Advanced Reproductive Age. Fertil Steril (2018) 109(4):728-34.e2. doi: 10.1016/ j.fertnstert.2017.12.024

30. Zhang J, Wang Y, Liu H, Mao X, Chen Q, Fan Y, et al. Effect of In Vitro Culture Period on Birth Weight After Vitrified-Warmed Transfer Cycles: Analysis of 4,201 Singleton Newborns. Fertil Steril (2019) 111(1):97-104. doi: 10.1016/j.fertnstert.2018.10.006

31. Zhang J, Liu H, Mao X, Chen Q, Fan Y, Xiao Y, et al. Effect of Body Mass Index on Pregnancy Outcomes in a Freeze-All Policy: An Analysis of 22,043 First Autologous Frozen-Thawed Embryo Transfer Cycles in China. BMC Med (2019) 17(1):114. doi: 10.1186/s12916-019-1354-1

Conflict of Interest: The authors declare that the research was conducted in the absence of any commercial or financial relationships that could be construed as a potential conflict of interest.

Publisher's Note: All claims expressed in this article are solely those of the authors and do not necessarily represent those of their affiliated organizations, or those of the publisher, the editors and the reviewers. Any product that may be evaluated in this article, or claim that may be made by its manufacturer, is not guaranteed or endorsed by the publisher.

Copyright $\odot 2022 \mathrm{Ni}$, Wan, Zhou, Kuang and Yu. This is an open-access article distributed under the terms of the Creative Commons Attribution License (CC BY). The use, distribution or reproduction in other forums is permitted, provided the original author(s) and the copyright owner(s) are credited and that the original publication in this journal is cited, in accordance with accepted academic practice. No use, distribution or reproduction is permitted which does not comply with these terms. 